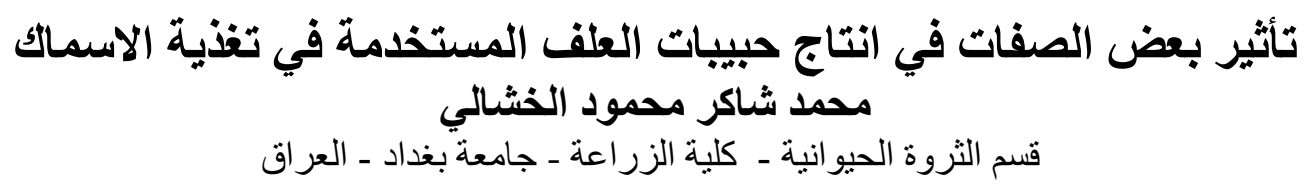

\title{
الخلاصة
}

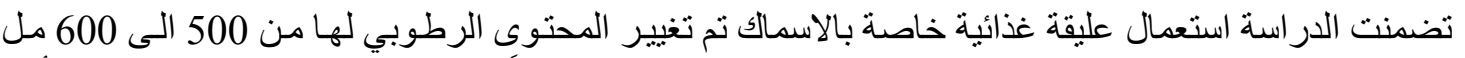

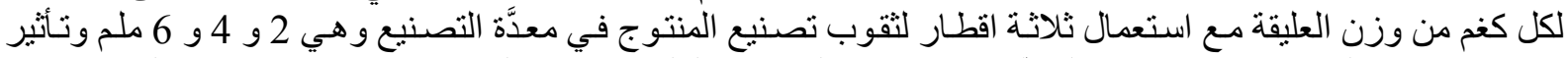

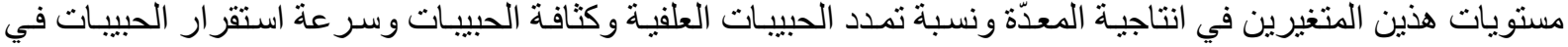

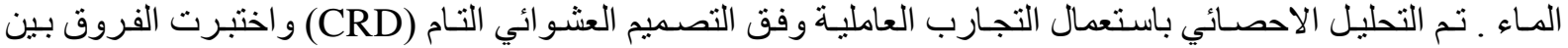

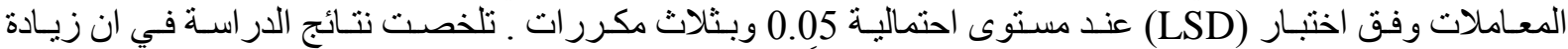

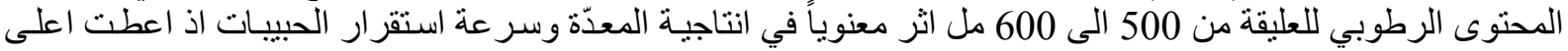

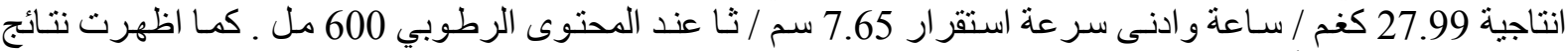

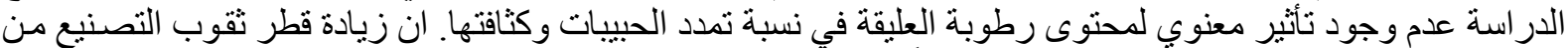

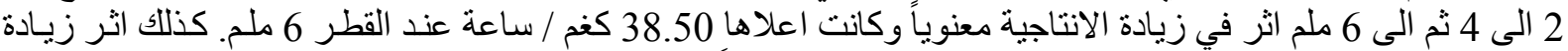

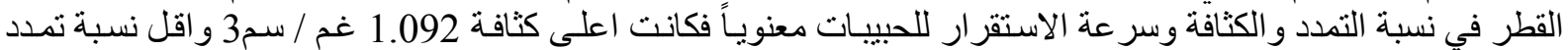

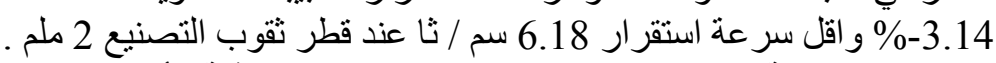
الكلمات المفتاحية :المحتوى الرطوبي, حبيبات العلف, تغذية الأسماك.

\section{Effect of Some Parameters in Pellets Production Used for Fish Feeding}

\section{Sh. M. Al-Khshali \\ Department of Animal Resources, College of Agriculture, University of Baghdad, Iraq Accepted in 5/1/2012 \\ Summary}

The study dealt with the usual fish feed used in fish farms by changing its moisture content from 500 to $600 \mathrm{ml} / \mathrm{kg}$ and using three different hole diameters $(2,4,6 \mathrm{~mm})$ in manufacturing the pellets. These two variables were studied concerns the effect on productivity and expansion ratio of the pellets, their density and its settling velocity in water, by using the factorial experiment under Completely Randomized Design (CRD) with three replicates and significant effect tests between treatments under (LSD) test at (0.05). The Results showed that with increase mash of diet moisture content from 500 to $600 \mathrm{ml}$ found significant effect in productivity and it provided maximum productivity $(27.99 \mathrm{~kg} / \mathrm{h})$ with moisture content $600 \mathrm{ml}$ and it significant effect in settle velocity and was the least settle velocity $(7.65 \mathrm{~cm} / \mathrm{s})$ at moisture $600 \mathrm{ml}$ and results study not found significant effect in expansion ratio and density for pellets with the changing the product holes diameter from 2 to 4 to $6 \mathrm{~mm}$ found significant effect in productivity it up was $(38.50 \mathrm{~kg} / \mathrm{h})$ at hole diameter 6 $\mathrm{mm}$ and also found significant effect in expansion ratio and density and settle velocity for pellets it was up the density $\left(1.092 \mathrm{gm} / \mathrm{cm}^{3}\right)$ and the least expansion ratio $(-3.14 \%)$ and the least settle velocity $(6.18 \mathrm{~cm} / \mathrm{s})$ at the holes diameter $2 \mathrm{~mm}$.

Keywords: Cyprinus carpio, Pellets , Feeding, Manufacture.

\section{المقدمة}

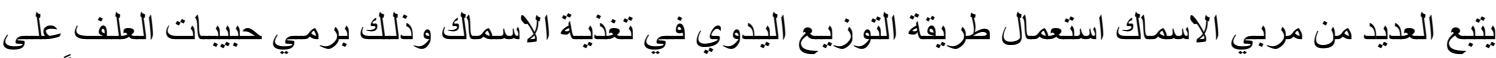

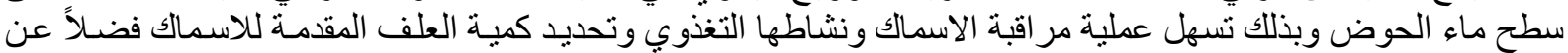

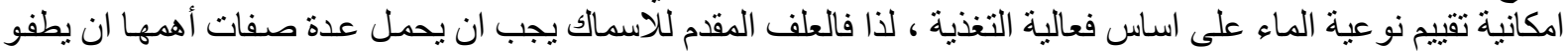

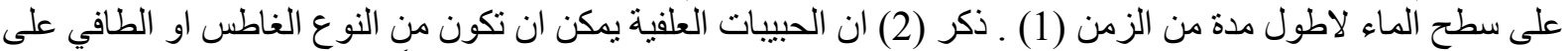

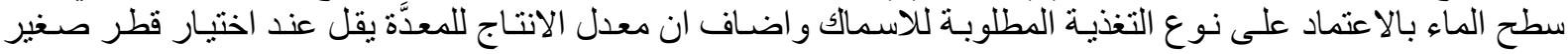


لثقوب التصنيع. واوضح (3) ان المحتوى الرطوبي للعليقة يؤثر في نوعيـة الحبيبات المنتجـة ومعدل انتاج الحبيبـات ـ كمـا

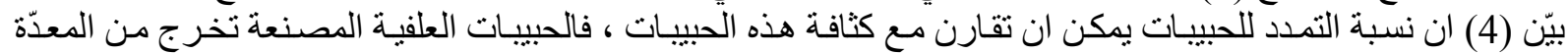

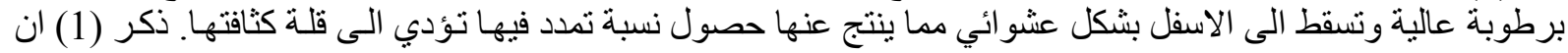

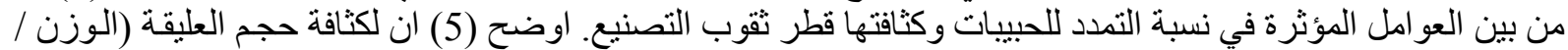

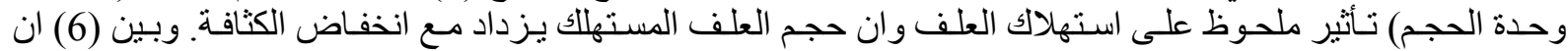

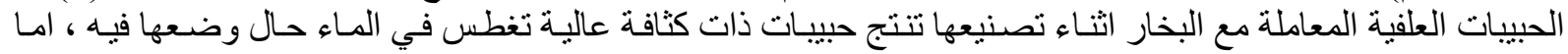

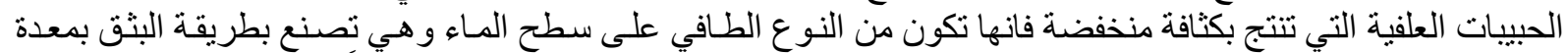

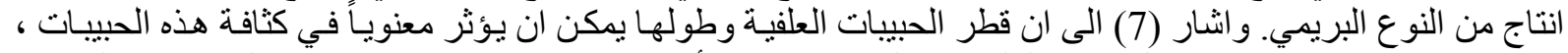

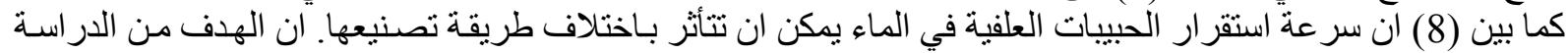

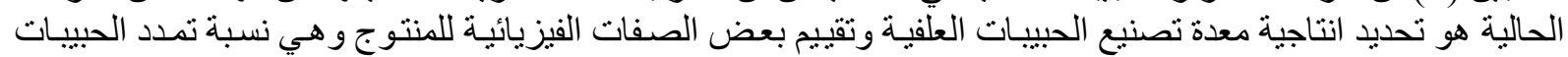

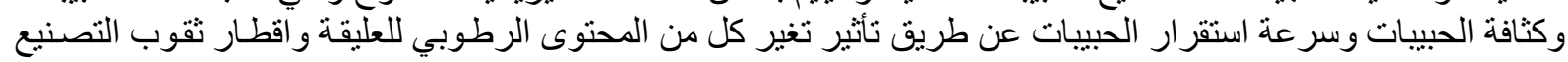
في المعدة.

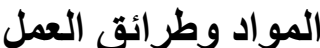

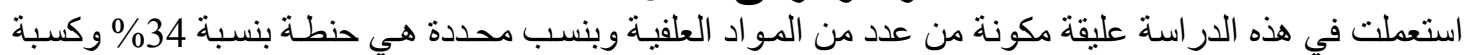
فول الصويا بنسبة 20\% وذرة صفر اء بنسبة 35\% وبروتين حيو اني بنسبة 10\% وخليط فيتامينات ومعادن بنسبة 1\%

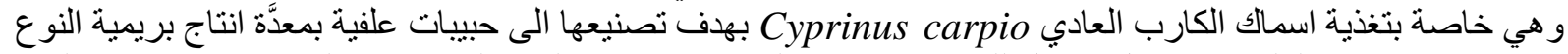

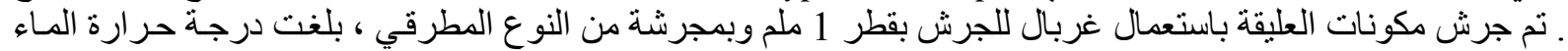

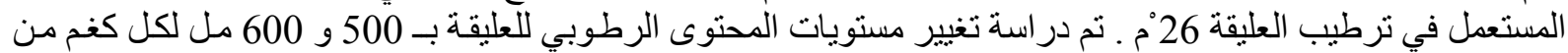

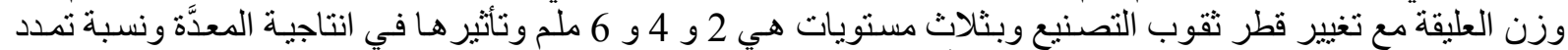

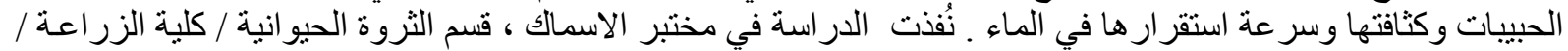

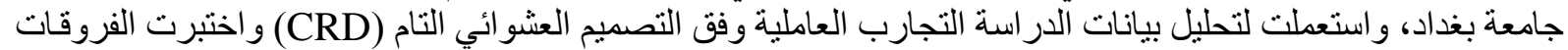

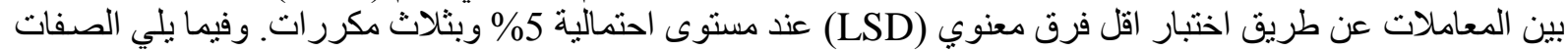

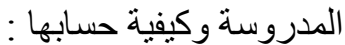

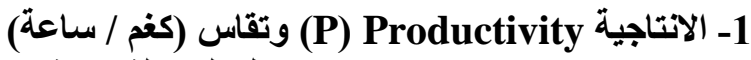
تم حسابها وفق (9) وباستعمال المعادلة الاتية :

$$
\begin{aligned}
& \text { الوزن الناتج (كغم) }
\end{aligned}
$$

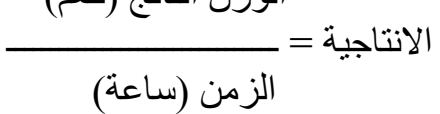

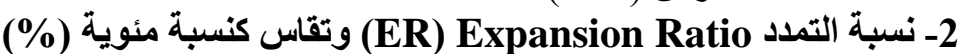

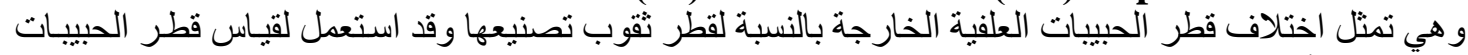

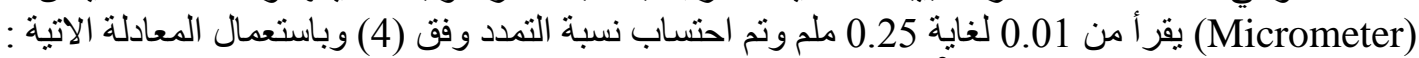

$$
\begin{aligned}
& \text { (قطر الحبيية العلفية) ملم }
\end{aligned}
$$

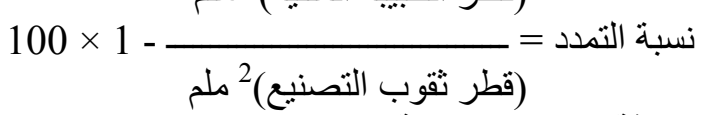

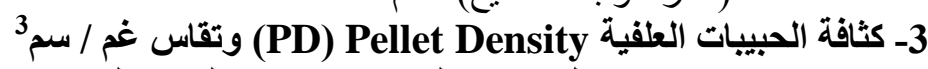

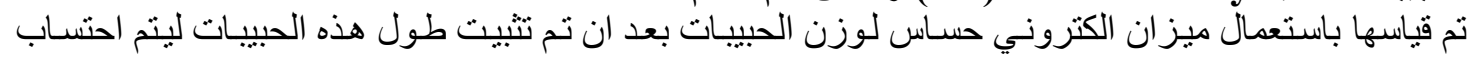
الكثافة وفق (4) وباستعمال المعادلة الاتية:

كتلة الحبيبة العلفية (غم)

$$
\text { طول الحبيبة (سم) × مساحة المقطع العرضي للحبيبة (سم²) }
$$

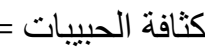

4- سرعة استقرار الحبيبات Settling Velocity

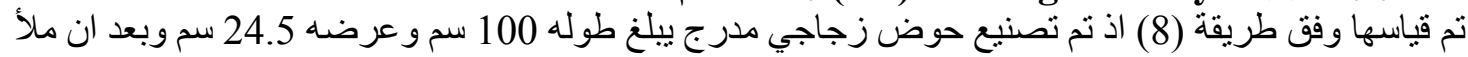

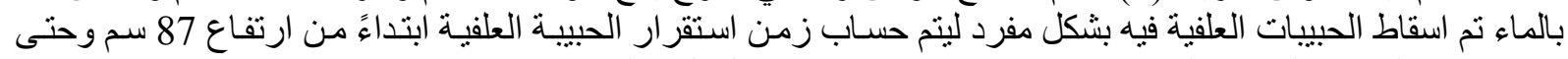
استقر ارها على قاع الحوض ليتم حساب سر عة الاستقر ار باستعمال المعادلة الاتية : (سم) 87

زمن الاستقرار (ثا) 


\section{النتائج والمناقشة}

1 - 1 الانتاجية (P) كفم / ساعة

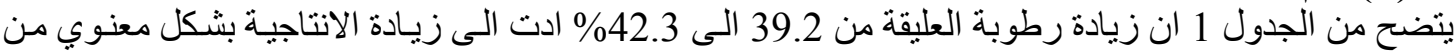

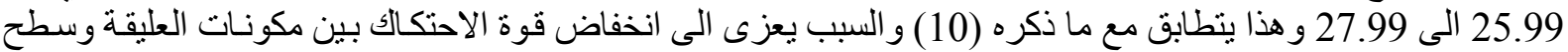

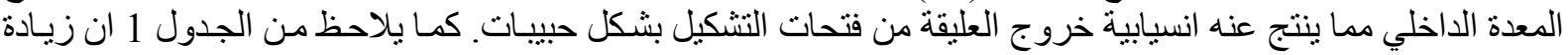

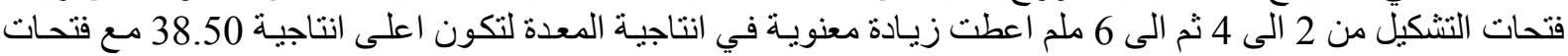

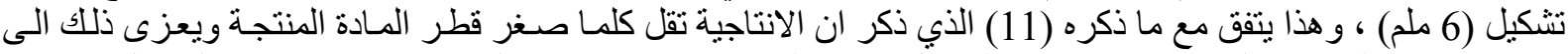

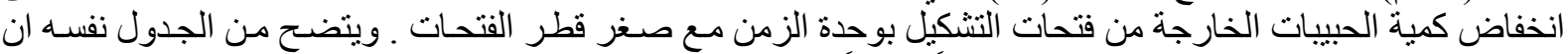

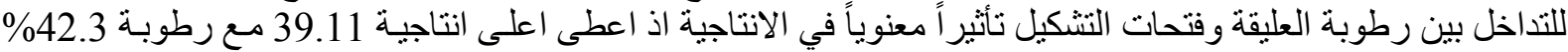

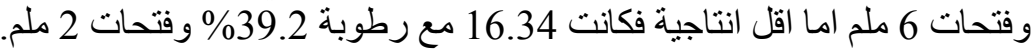

جدول (1) تأثير رطوبة العليقة وفتحات التثكيل وتداخلهما في الانتاجية (كفم/ ساعة)

\begin{tabular}{|c|c|c|c|c|}
\hline \multirow{2}{*}{ الرطوية معدل } & \multicolumn{3}{|c|}{ فَتحات الثشكيل (مام) } & \multirow{2}{*}{ رطوبة العليقة } \\
\hline & 6 & 4 & 2 & \\
\hline $25.99 \mathrm{~b}$ & $37.90 \mathrm{~b}$ & $23.72 \mathrm{D}$ & $16.34 \mathrm{f}$ & 39.2 \\
\hline \multirow[t]{4}{*}{27.99 a } & $39.11 \mathrm{a}$ & $26.06 \mathrm{c}$ & $18.79 \mathrm{e}$ & 42.3 \\
\hline & $38.50 \mathrm{a}$ & $24.89 \mathrm{~b}$ & $17.57 \mathrm{c}$ & معدل الفتحات \\
\hline & \multicolumn{4}{|c|}{ اقل فرق معنوي عاند مستوى 5\% } \\
\hline & & الرطوبة & التداخل : 0.79 & القطر : 0.56 \\
\hline
\end{tabular}

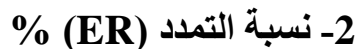

يلاحظ من الجدول 2 ان زيادة محتوى رطوبة العليقة من 500 الى الى 600 مل مل لم يعطي تأثير أ معنوياً على نسبة

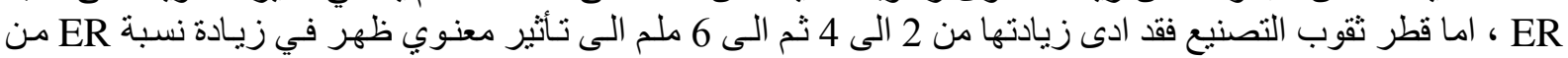

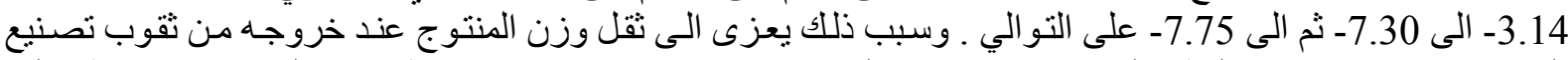

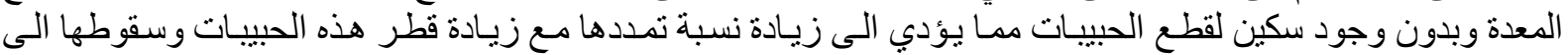

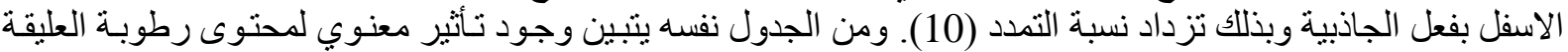

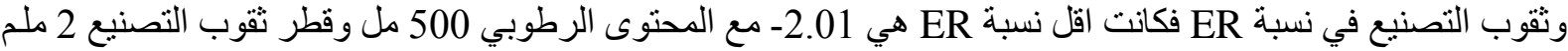

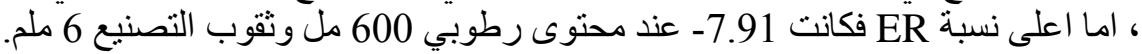

جدول (2) تأثير محتوى رطوبة العليقة وقطرثقوب التصنيع والتذاخل بينهما في نسبة التمدد

\begin{tabular}{|c|c|c|c|c|}
\hline معال & \multicolumn{3}{|c|}{ مُشقب الآتصنيع (مام) } & \multirow{2}{*}{ محتوى رطوبة العايقة: } \\
\hline الرطوبة & 6 & 4 & 2 & \\
\hline$-5.62 a$ & $-7.60 b$ & $-7.24 b$ & $-2.01 \mathrm{a}$ & 500 \\
\hline \multirow[t]{4}{*}{$-6.52 a$} & $-7.91 \mathrm{~b}$ & $-7.37 \mathrm{~b}$ & $-4.27 \mathrm{a}$ & 600 \\
\hline & $-7.75 b$ & $-7.30 \mathrm{~b}$ & $-3.14 \mathrm{a}$ & معدل قطر الثثقوب \\
\hline & & & $\% 5$ & اقل فرق معنوي عند مسن \\
\hline & & الرطوبة & التذاخل : 2.73 & القطر : N.S \\
\hline
\end{tabular}

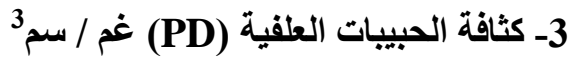

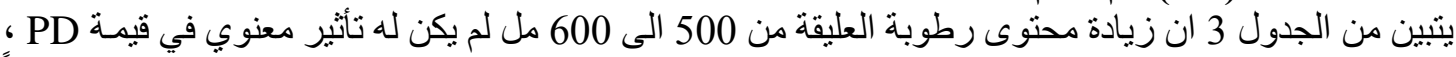

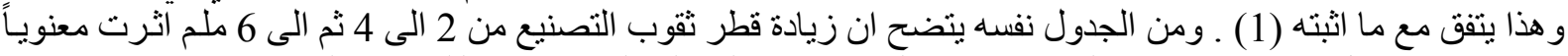

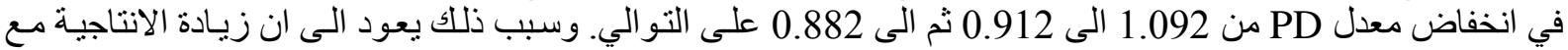

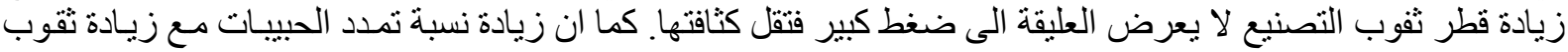

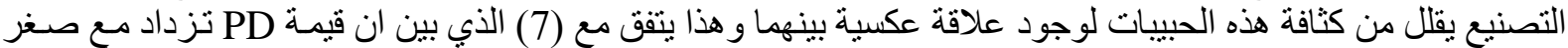

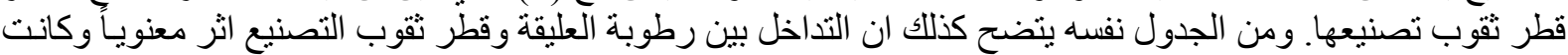

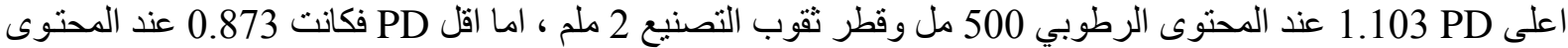
الرطوبي 600 مل وقطر ثقوب التصنيع 6 ملم. 


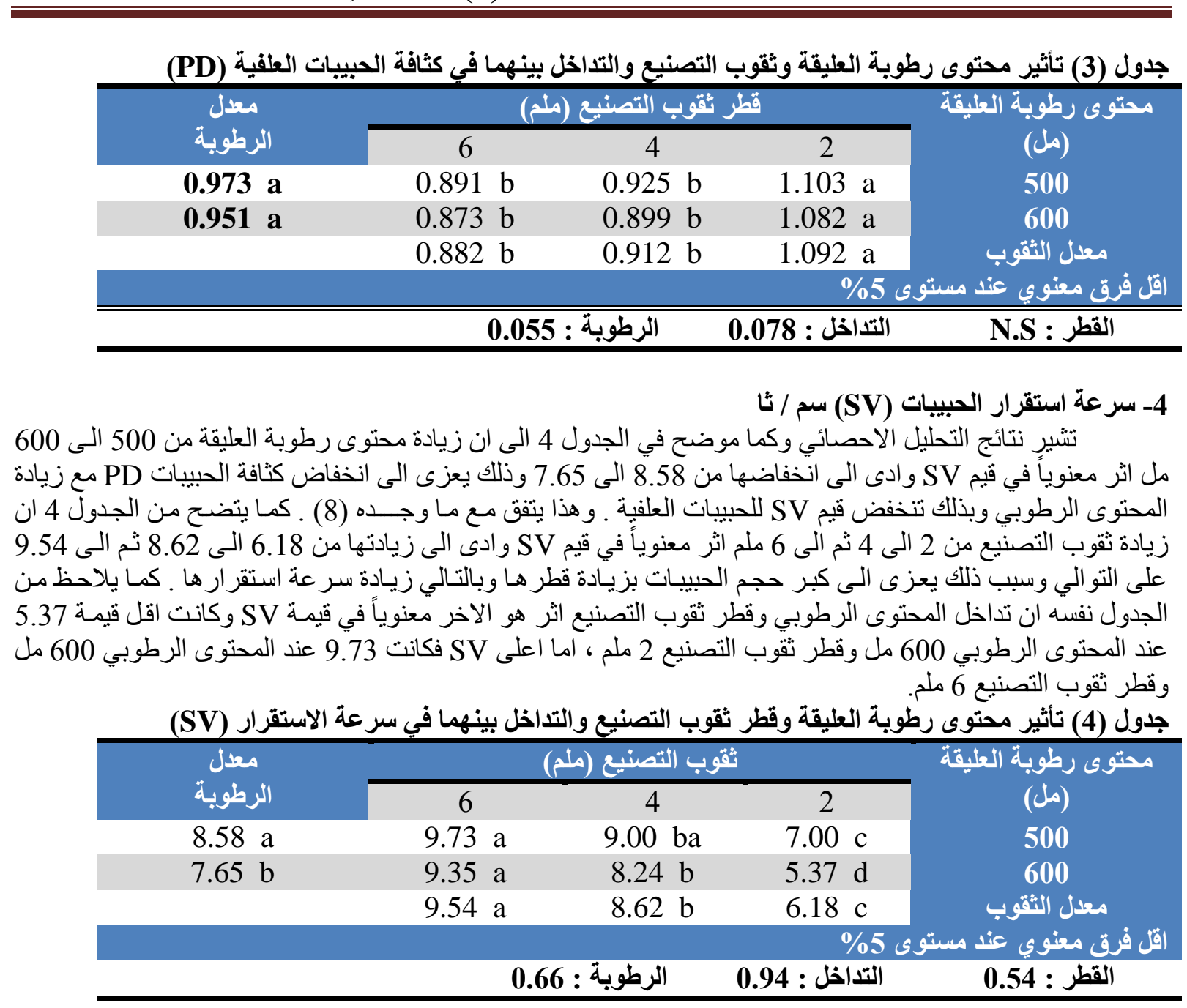

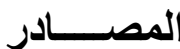

1. Rolfe, LA.; Huff, HE. and Hsieh, F. ( 2000). The effect of processing conditions on the quality of extruded catfish feed. Amer. Soc. Agric. Eng., 43 (6): 1737-1743.

2. New, MB. (1987). Feed and feeding of fish and shrimp. Food and Agric Org of the United Nations, (FAO) p: 234.

3. Herrman, TJ. and Loughin, T. (2003). Processing and shelf life performance of feed manufactured from high-moisture corn. Amer. Soc. Agric. Eng., 46 (3): 697-703.

4. Misra, C K.; Sahu, NP. and Jain, KK. (2002). Effect of extrasion processing and steam pelleting diets on pellet durability, water absorption and physical response of macrobrachium rosenbergii, 15 (9) : 1354-1358. India.

5 ـ بن عامر ، محمد السنوسي وحسن محمد واحمد الحاج. (1997). اساسيات تغذية واعلاف الحيوان ـ الدجلد الثالث ، منشورات

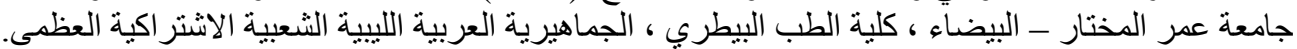

6. Lovell, T. (1989). Nutrition and feeding of fish. Van Nostr and rein pub New York, USA., P: 248.

7. Rokey, GR.; Strathman, and Plattner, B. (2002). Pelleted livestock feed production. Process description, Wenger MFG INC, p: 185.

8. Rout, RK. and Bandyopadyay, S. (1998). A comparative study of shrimp feed pellets processed through cooking extruder and meat mincer. Indian instit of techn, Kharagpur, 71-79.

9. Handerson, SM. and Perry, RL. (1955). Agricultural process engineering. Jhon Wiley and Sons. INC. p: 192.

10. Fairfield, DA. (2003). Pelleting for profit - part 2. National grain and feed association, 54 (7):114. 11 ـ محمد علي ، لطفي حسين وتوفيق فهمي دميان(1988). معدات مكنتة الانتاج الحيو اني ـ جامعة بغداد ، وزارة التعليم العالي . 